\title{
Dispositivo Interactivo Luminoso como Recurso Didáctico para la Mejor Comprensión del Concepto Ácido-Base en Química General
}

\author{
Silvana R. Matkovic, Laura E. Briand \\ Centro de Investigación y Desarrollo en Ciencias Aplicadas Dr. Jorge J. Ronco CINDECA-CCT \\ La Plata-CONICET- UNLP. Calle 47 No 257, B1900AJK, La Plata, Buenos Aires-Argentina. \\ (e-mail: matkovic@quimica.unlp.edu.ar, briand@quimica.unlp.edu.ar)
}

Recibido Nov. 02, 2011; Aceptado Dic. 13, 2011; Versión final recibida Ene. 06, 2012

\begin{abstract}
Resumen
Se propone y construye un recurso didáctico para ayudar al alumno a comprender en mejor forma el concepto ácido-base en química general, en particular el proceso de cambio de color observado al interaccionar un indicador ácido-base con una sustancia ácida o alcalina. La propuesta consiste en la construcción de un dispositivo sencillo, interactivo y luminoso. El mismo, consta de dos partes que al ponerse en contacto cierran un circuito que enciende una luz, por esto presenta un color si posee sus dos partes constitutivas unidas y sufre una modificación del color si las mismas están separadas. Esta propuesta también involucra una parte experimental donde sustancias de uso diario se ponen en contacto con colorantes naturales. El uso del dispositivo luminoso de tipo interactivo constituye un recurso didáctico que permite correlacionar a nivel macroscópico la interacción entre especies químicas y el cambio de color que experimenta un indicador ácidobase.
\end{abstract}

Palabras clave: enseñanza de la química, ácido-base, $\mathrm{pH}$, indicadores ácido-base

\section{Interactive Lighting Device as a Teaching Resource for the Better Comprehension of the Acid-Base Concept in General Chemistry}

\begin{abstract}
A teaching resource is constructed with the aim of helping student to better comprehend the acidbase concept in general chemistry, in particular the process of color change that is observed when an acid-base indicator reacts with acid or basic species. The proposal involves an interactive lighting device easy to build. The device lights when its two parts are in contact closing an electric circuit. Therefore it has a certain color when both parts are in contact that differs from the color of the when they are apart. Additionally, the contact of natural dyes with everyday domestic substances is described as a complementary experimental section. The interactive lighting device is a teaching resource that allows correlating the interaction of a chemical species with the change of color of an acid-base indicator.
\end{abstract}

Keywords: chemistry teaching, acid-base, $\mathrm{pH}$, acid-base indicators 


\section{INTRODUCCIÓN}

Los ácidos y las bases son hoy sustancias ampliamente conocidas en la química. Desde los tiempos antiguos el vinagre se obtenía de la sidra de manzana y del vino. La palabra latina que significa vinagre, acetum, está relacionada muy de cerca con otra palabra latina, acidus, de donde proviene la palabra ácido. Desde hace mucho tiempo se ha sabido que los líquidos de sabor agrio contienen ácidos y las sustancias que son básicas o alcalinas tienen un sabor amargo. Cabe destacar que el concepto de ácido, ya aparece en el manuscrito indio Rasarnava, 1200 años A.C. Las bases eran conocidas como álcalis, debido a que el más conocido (carbonato potásico) se extraía de las cenizas de la planta Kali (la primera referencia se da en la obra de Abu Mansur Monafir, siglo $X$ d.C.).

Existen referencias más antiguas, ya que en las tablas sumerias las cenizas vegetales eran conocidas como Te-Gaz. En 1664, Boyle escribió "The Experimental History of Colours". En ella se inicia el reconocimiento de ácidos y bases a través de los cambios de color de extractos de plantas. A partir de Boyle una de las formas de indicar la presencia de un ácido fue utilizar como indicador el cambio de color del jarabe de violetas y a partir de ese momento nacen los indicadores químicos. En el primer diccionario de Química publicado por Macquer en 1766, aparece como definición de los álcalis: sustancias que "vuelven verde el jarabe de violetas". El término base, surge a mediados del siglo XVIII, y se debe al químico francés Rouelle, ya que eran la base de la formación de las sales al combinarse con los ácidos. En 1671, Duclós llama "turnesol" (litmus), a un indicador extraído de líquenes, que le da un gran resultado. Casi cien años después, James Watt, el inventor de la máquina de vapor y nominador del caballo de vapor como unidad de potencia, descubre que la lombarda (col roja) es uno de los mejores indicadores naturales (Partington, 1962).

Desde el trabajo realizado por Boyle y posteriores interpretaciones del fenómeno fue necesaria una evolución que llevó casi 200 años hasta el primer modelo científico que explicó la naturaleza de ácidos, bases y las reacciones que involucran a los mismos. Como puede verse a través del breve desarrollo histórico presentado que va desde la definición de la existencia de los ácidos y bases a través de distintas caracterizaciones de los mismos, el concepto ácido-base es un concepto complejo de transmitir. En sí, la química es una ciencia al mismo tiempo muy concreta y muy abstracta. Los fenómenos que estudia nunca son fáciles de caracterizar, pues la relación entre lo que se observa y lo que hoy se sabe que sucede no es en ningún caso evidente (Izquierdo et al., 2007). Un modelo científico es una entidad abstracta, una representación simplificada de un hecho, objeto, fenómeno, realizada con la finalidad de describir, explicar y predecir. En este sentido, los modelos científicos constituyen uno de los principales productos de la ciencia (Raviolo et al., 2010). Por esta razón, las analogías y las metáforas juegan un papel central en el proceso educativo. Crean puentes entre lo conocido y lo desconocido uniendo realidades que hasta ese momento eran extrañas. Por esto, la utilización de diversos recursos didácticos en el aula permite un mayor acercamiento al modelo científico. Actúan de nexo entre los conocimientos previos de los alumnos, la observación experimental y los modelos científicos. Éstos últimos son los que buscamos que el alumno internalice y apropie, a fin de formar parte de su conocimiento formal.

La perspectiva pedagógica de la enseñanza a través de analogías y metáforas, no centra su atención en el "cambio conceptual" ni en la aplicación del "método científico", sino en la explicitación que el estudiante hace de los hechos observados a través de distintos lenguajes, es decir, en la construcción de modelos explicativos que se enriquecen con las nuevas experiencias y el intercambio de puntos de vista entre los miembros del grupo. Por ello, la hipótesis en la construcción de modelos apunta no a la superación empírica de un modelo científico por otro, sino a la integración jerárquica que el estudiante va haciendo del conocimiento cotidiano y el científico, considerando que también aprenden a diferenciar e integrar diversos tipos de explicaciones, y no sólo eso, sino que reconocen los contextos en los que son relevantes (Pozo y Gómez-Crespo, 1998). El objetivo de este trabajo apunta a la utilización de un dispositivo luminoso interactivo (se denominará DLI a lo largo del manuscrito) como recurso didáctico. Se propone utilizarlo para ayudar al alumno a correlacionar su funcionamiento con el proceso de cambio de color observado 
al interaccionar un indicador ácido-base con una sustancia ácida o alcalina, y utilizarlo para introducir el modelo científico.

\section{METODOLOGÍA}

En un principio se propone introducir la noción de indicador ácido-base, incentivando la observación de que a nuestro alrededor tanto frutas como verduras poseen su color gracias a pigmentos naturales y que dicho color puede variar de acuerdo a la acidez o basicidad del medio acuoso en el que se encuentren. Asimismo el docente, explicará que estos colores se dan por la existencia de sustancias polifenólicas llamadas antocianinas y antoxantinas que son solubles en solventes polares, y por esto pueden extraerse de los productos naturales con alcohol o bien agua fría o caliente. En este punto se invita al docente a promover el entusiasmo e interés de los alumnos a fin de que experimenten y elijan flores o frutos y que intenten extraer los distintos pigmentos (Fanis, 2008). Los pigmentos extraídos (también se pueden utilizar té y vino) se hacen interaccionar con sustancias conocidas por los alumnos como una bebida gaseosa, limpia vidrios, vinagre blanco, desengrasante de cocina y solución jabonosa. Así se reconoce el carácter ácido o básico tomando como color ácido el que se obtiene al interaccionar la solución colorante con un compuesto ácido reconocido por los alumnos como por ejemplo el jugo de limón o el vinagre.

Luego de la observación experimental se propone la utilización del DLI a fin interpretar los cambios de color observados en los experimentos descriptos anteriormente. El mismo, consta de dos partes que al ponerse en contacto cierran un circuito que enciende una luz, por esto presenta un color si posee sus dos partes constitutivas unidas y sufre una modificación del color si las mismas están separadas. En este contexto, se propone hacer una analogía donde el color ácido $(H I n)$ queda representado por el DLI con sus dos partes componentes en contacto. En contraste, el color básico $(I n)$ y el protón $\left(H^{+}\right)$están representados cada uno de ellos por las dos partes separadas del DLI. Finalmente, el docente correlacionará la analogía con la ecuación química (1) que se presenta a continuación.

\section{$\mathrm{HIn} \longleftrightarrow \mathrm{In}^{-}+\mathrm{H}^{+}$ \\ Color ácido Color básico}

En la Figura 1 se muestran los materiales necesarios para el armado del DLI, los cuales son: un recipiente transparente de color con tapa; una lámpara tipo led de color contrastante con respecto al recipiente, una pila botón de $3 \mathrm{~V}$, papel aluminio, cables y cinta aisladora. En la Figura 2 se muestra la preparación del led y de la conexión correspondiente a la tapa del recipiente, en esta instancia se unen los cables a los contactos del led envolviendo los empalmes con cinta aisladora y cuidando que no hagan contacto entre sí. Se enroscan los cables y se pasan a través del agujero de la tapa dosificadora. En la Figura 3 puede observarse el detalle del armado del circuito. Los cables deben disponerse de tal forma que entren en contacto con los polos de la pila cuando las dos partes componentes del DLI se unen.

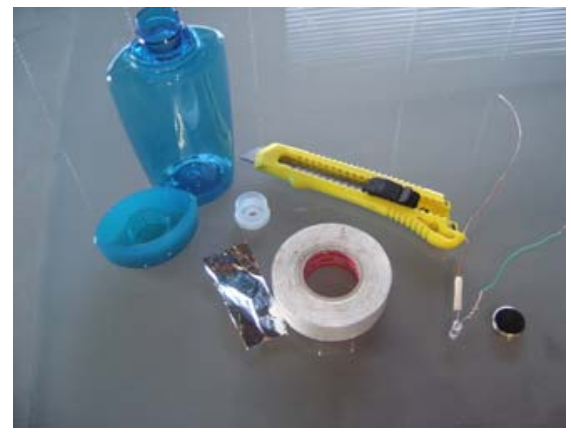

Fig. 1. Materiales a utilizar en la construcción del dispositivo.

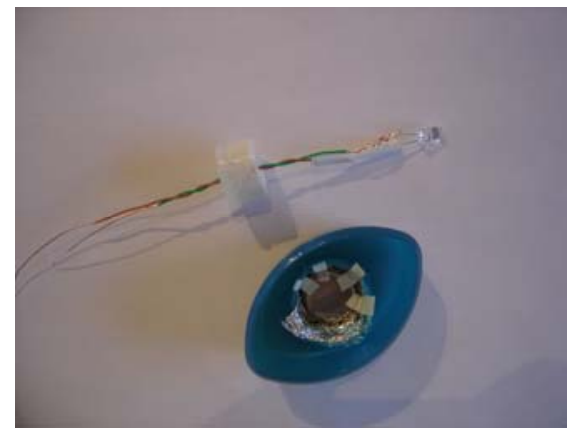

Fig. 2. Circuito del led, cables y la pila.

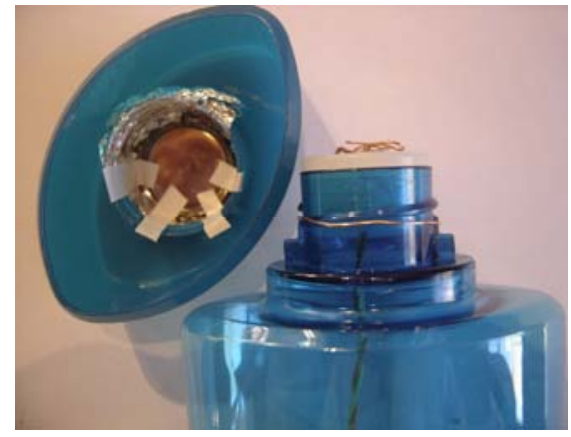

Fig. 3. Armado final del dispositivo. 


\section{RESULTADOS}

En este trabajo se presenta un DLI para usarse como analogía en el cambio de color de las especies químicas y a su vez generar un puente a fin de introducir el modelo científico que se utiliza en el ámbito académico para explicar la naturaleza ácido-base de los indicadores. En el desarrollo de la propuesta se trata de que los alumnos analicen situaciones concretas, cercanas al entorno conocido por ellos y de esta manera introducir en forma gradual entidades menos familiares y más abstractas -generadas en el marco de la ciencia- para explicar las situaciones experimentales seleccionadas. Así de esta manera ir de lo simple a lo complejo.

Este recurso didáctico permitiría introducir el concepto de ácido de Brönsted-Lowry, la presencia del ácido y base conjugada, y la ecuación química que representa la disociación de un indicador de carácter ácido. Por otro lado podría introducirse el concepto de equilibrio químico relacionado con la preponderancia de un color sobre otro. Así si hubiera más protones (representados por la tapa del DLI) requeriría más cantidad de cuerpos del DLI (especie Iñ) las que se asociarían y harían predominar el color ácido, aunque no desaparecen las especies en ningún momento.

\section{CONCLUSIONES}

Con base en los resultados y la discusión presentada, se pueden extraer las siguientes conclusiones principales: 1) el dispositivo presenta un color si sus dos partes constitutivas están unidas y otro color si las mismas están separadas; 2) el funcionamiento del dispositivo lumínico interactivo permite correlacionar a nivel macroscópico la interacción entre especies químicas y el cambio de color que experimenta un indicador ácido-base; 3) la idea de cambio de color de una especie química de acuerdo al $\mathrm{pH}$ del medio puede reforzarse en el laboratorio a través del contacto de sustancias extraídas de frutas y/o flores (que son indicadores naturales) y sustancias de uso cotidiano como lavandina, vinagre, entre otras; 4) finalmente relacionando las observaciones realizadas en el laboratorio y el funcionamiento del dispositivo lumínico interactivo se presenta el modelo dado por ecuaciones químicas específicas.

La versatilidad del recurso didáctico propuesto permite su utilización en los niveles educativos secundario y universitario dependiendo de la profundidad con la que el docente desarrolle el tema.

\section{AGRADECIMIENTOS}

A la Secretaría de Extensión de la Facultad de Ciencias Exactas de la Universidad Nacional de La Plata (FCE - UNLP) por el apoyo económico recibido.

\section{REFERENCIAS}

Fanis, L.N., A colorful connection for your classroom, Journal of Chemical Education, Vol. 85 (9) 1172-1174 (2008).

Izquierdo, M., Caamaño, A. y M. Quintanilla, Investigar en la enseñanza de la química. Nuevos horizontes: contextualizar y modelizar, Bellaterra: Servei de Publicacions-Universitat Autònoma de Barcelona, $1^{\text {ra }}$ edición, Cap. 6, pp.142-164, Barcelona, España (2007).

Partington, J.R., A History of Chemistry, 4 vols., Macmillan \& Co. Ltd., Londres, Inglaterra (19611970).

Pozo, J.I. y M.A. Gómez Crespo, Aprender y enseñar ciencia, $1^{\text {ra }}$ edición, Morata, España (1998).

Raviolo, A., Ramírez, P., López, E.A. y A. Aguilar, Concepciones sobre el conocimiento científico y los modelos científicos: Un estudio preliminar, Rev. Formación Universitaria, Vol. 3(5), 29-36 (2010) 